\title{
BMJ Open Effectiveness of home or hospital initiation of treatment at diagnosis for children with type 1 diabetes (DECIDE trial): a multicentre individually randomised controlled trial
}

John W Gregory, ${ }^{1}$ Julia Townson (1) , ${ }^{2}$ Sue Channon, ${ }^{2}$ David Cohen, ${ }^{3}$ Mirella Longo, ${ }^{4}$ Justin Davies, ${ }^{5}$ Nicola Harman, ${ }^{6}$ Kerenza Hood, ${ }^{2}$ Timothy Pickles, ${ }^{2}$ Rebecca Playle, ${ }^{2}$ Tabitha Randell, ${ }^{7}$ Michael Robling (I) ,, Morro Touray, ${ }^{8}$ Nicola Trevelyan, ${ }^{5}$ Justin Warner, ${ }^{9}$ Lesley Lowes ${ }^{10}$

To cite: Gregory JW, Townson J, Channon S, et al. Effectiveness of home or hospital initiation of treatment at diagnosis for children with type 1 diabetes (DECIDE trial): a multicentre individually randomised controlled trial. BMJ Open 2019;9:e032317. doi:10.1136/ bmjopen-2019-032317

- Prepublication history and additional material for this paper are available online. To view these files, please visit the journal online (http://dx.doi org/10.1136/bmjopen-2019032317).

Received 12 June 2019 Revised 23 October 2019 Accepted 04 November 2019

Check for updates

(c) Author(s) (or their employer(s)) 2019. Re-use permitted under CC BY-NC. No commercial re-use. See rights and permissions. Published by BMJ.

For numbered affiliations see end of article.

Correspondence to

Dr Julia Townson;

townson@cf.ac.uk

\section{ABSTRACT}

Objective To determine whether, in children with newly diagnosed type 1 diabetes who were not acutely unwell, management at home for initiation of insulin treatment and education of the child and family, would result in improved clinical and psychological outcomes at 2 years postdiagnosis. Design A multicentre randomised controlled trial (January 2008/0ctober 2013).

Setting Eight paediatric diabetes centres in England, Wales and Northern Ireland.

Participants 203 clinically well children aged under 17 years, with newly diagnosed type 1 diabetes and their carers.

Intervention Management of the initiation period from diagnosis at home, for a minimum of 3 days, to include at least six supervised injections and delivery of pragmatic educational care.

Main outcome measures Primary outcome was glycosylated haemoglobin ( $\mathrm{HbA1c}$ ) concentration at 24 months postdiagnosis. Secondary outcomes included coping, anxiety, quality of life and use of NHS resources.

Results 203 children, newly diagnosed, were randomised to commence management at home $(n=101)$ or in hospital $(n=102)$. At the 24 month primary end point, there was one withdrawal and a follow-up rate of 194/202 (96\%). Mean $\mathrm{HbA1c}$ in the home treatment arm was $72.1 \mathrm{mmol} / \mathrm{mol}$ and in the hospital treated arm $72.6 \mathrm{mmol} / \mathrm{mol}$. There was a negligible difference between the mean $\mathrm{HbA1c}$ levels in the two arms adjusted for baseline $(1.01,95 \% \mathrm{Cl} 0.93$ to 1.09). There were mostly no differences in secondary outcomes at 24 months, apart from better child selfesteem in the home-arm. No home-arm children were admitted to hospital during initiation and there were no adverse events at that time. The number of investigations was higher in hospital patients during the follow-up period. There were no differences in insulin regimens between the two arms.

Conclusions There is no evidence of a difference between home-based and hospital-based initiation of care in children newly diagnosed with type 1 diabetes across relevant outcomes.

\section{Strengths and limitations of this study}

- The largest multicentre randomised controlled trial to test the effectiveness of home or hospital initiation of treatment of children newly diagnosed with type 1 diabetes.

- The only adequately powered trial to test effectiveness of the location of care at diagnosis of type 1 diabetes in childhood.

- Inclusion of both teaching and district general hospitals, in England, Wales and Northern Ireland.

- Pragmatic approach to the clinical and educational care delivered during the initiation period to reflect differences in practice.

- There was a change in practice regarding insulin regimes in participating centres during the course of the trial.

Trial registration number ISRCTN78114042.

\section{INTRODUCTION}

The initial management of childhoodonset type 1 diabetes (T1D) is invasive and complex, requiring extensive support from diagnosis. Optimal blood glucose control is essential to minimise risk of life-changing complications but is difficult to achieve, placing young people at an unacceptably high risk of developing microvascular and macrovascular complications in later life. ${ }^{1}$ In addition, the emotional cost of T1D to the individual is significant, and a substantial long-term adverse psychological response to a diagnosis of T1D in childhood is reported in both children and parents/carers, particularly mothers. ${ }^{2}$ Although parents experience a grief response to diagnosis similar to that associated with bereavement, they report 


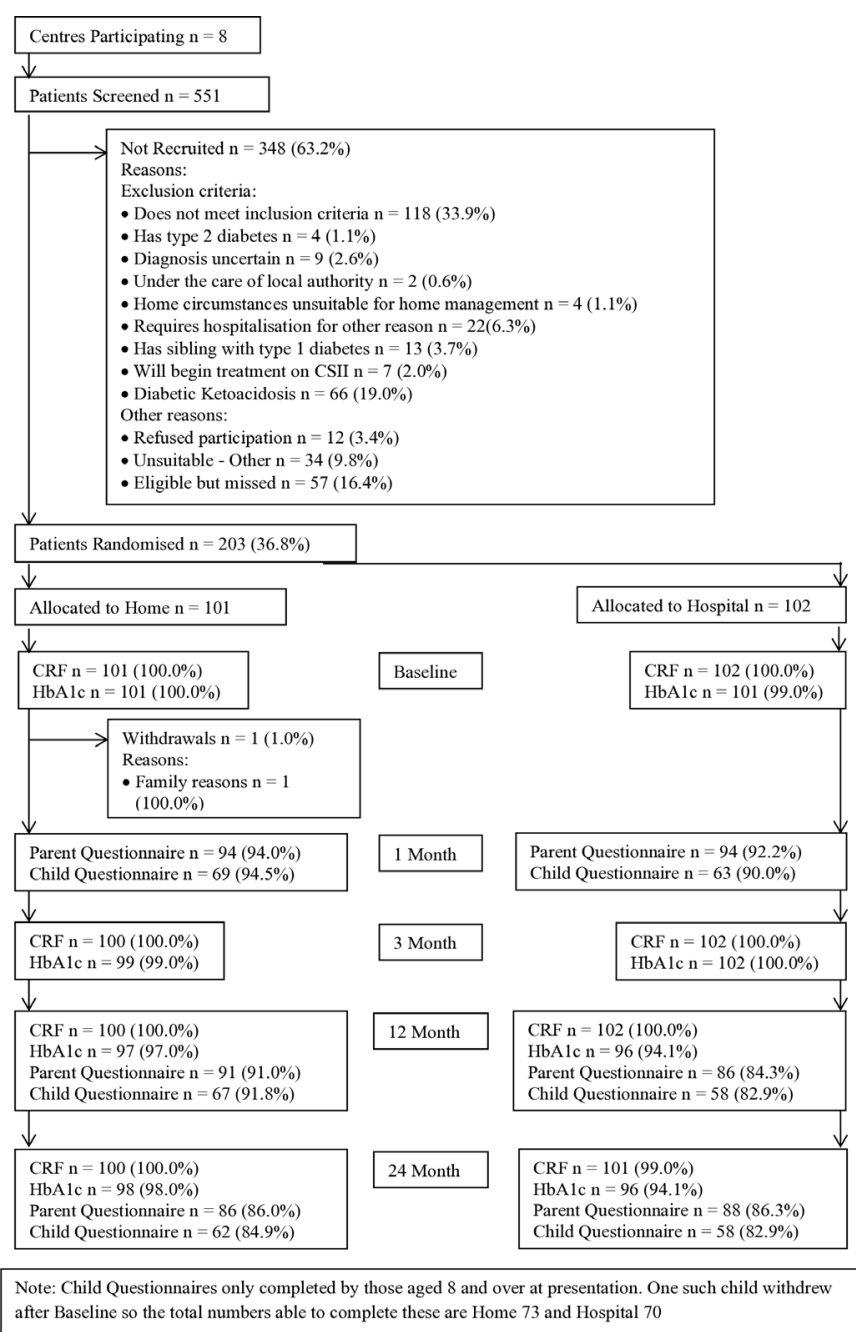

Figure 1 Participant flow diagram. CSII, continuous subcutaneous insulin infusion; CRF, Case Report Form; $\mathrm{HbA1c}$, glycosylated haemoglobin.

positive experiences of having their child managed at home from diagnosis, ${ }^{3}$ allowing them to deal with situations that occurred within the framework of their everyday lives. The relative normality of this helped them feel more 'in control' of the situation, enabling them to cope more effectively and feel less anxious.

There is a global trend to move clinical care from hospital into the community, reflecting concerns about healthcare costs, limited availability of hospital beds and the need to promote more integrated healthcare delivery to improve the quality and patient experience of care. ${ }^{45}$ However, community-based services are not necessarily cheaper than hospital care in the short term, and any particular model or initiative needs evaluation of transferability and adaptability into a specific healthcare system, safety, cost effectiveness and acceptability to patients and their families.

There is little agreement worldwide about where best to manage young people at diagnosis with practices varying from minimal hospitalisation with largely out-patient-based management in the UK, USA and Australia to admission for several weeks in Europe and Japan. ${ }^{6-8}$ Although hospitalisation is necessary if children are severely dehydrated or ketoacidotic, over half are relatively well at diagnosis, do not require intravenous therapy and stabilisation at diagnosis may not require hospitalisation. ${ }^{7910}$ It has been claimed that home management is a safe, effective alternative to hospitalisation at diagnosis ${ }^{311-14}$ but studies evaluating hospitalisation or home management at diagnosis are limited by their often retrospective nature and lack of power. ${ }^{615}$ A systematic review concluded that studies in this area were generally of low quality. It suggested that home-based management at diagnosis does not lead to disadvantaged metabolic control or other adverse outcomes and there was no difference in overall costs. A large well-designed randomised controlled trial was therefore required to investigate whether there are significant differences in short-term and long-term outcomes between the two approaches. ${ }^{6}$

We hypothesised that children who had their initial treatment and education managed at home would have better clinical and psychological outcomes than those managed in hospital. The aim of The Delivering Early Care in Diabetes Evaluation (DECIDE) trial, therefore, was to investigate over 2 years from diagnosis, the effect of initiating treatment at home or in hospital by assessing and comparing glycaemic control, in children with T1D aged under 17 years and to assess use of NHS resources during the initiation (days 0-3 after diagnosis) and subsequent follow-up periods. Cost-effectiveness and cost consequences analyses will be reported separately.

\section{METHODS}

A full description of the trial methods has been published $^{16}$ and can be accessed at http://www.biomedcentral.com/1471-2431/11/7. In brief, this was an individually randomised controlled trial at eight UK paediatric diabetes centres $(n=8)$ in NHS secondary care in England, Northern Ireland and Wales. Participating centres were Belfast, Cambridge, Cardiff, Hull, Liverpool, Newcastle, Nottingham and Southampton. Criteria for centre selection included a minimum of 30 newly diagnosed children per year and geographical placement to try and achieve diversity in socioeconomic parameters across included sites. Each centre comprised at least one consultant paediatrician with an interest in diabetes, a paediatric diabetes nurse and a paediatric dietitian in addition to other multidisciplinary team members.

\section{Participants}

Children and their carers were eligible to participate if the child was aged $<17$ years, newly diagnosed with T1D and if the child and parent/carer were able to complete study materials and give informed assent (child aged 8-12 years) and consent/assent (parent/carer and child aged 13-17 years).

Exclusion criteria were:

- Ketoacidosis requiring intravenous fluids and insulin.

- Children requiring hospitalisation for reasons other than their diagnosis of T1D. 
Table 1 Baseline characteristics of participants by arm and in total

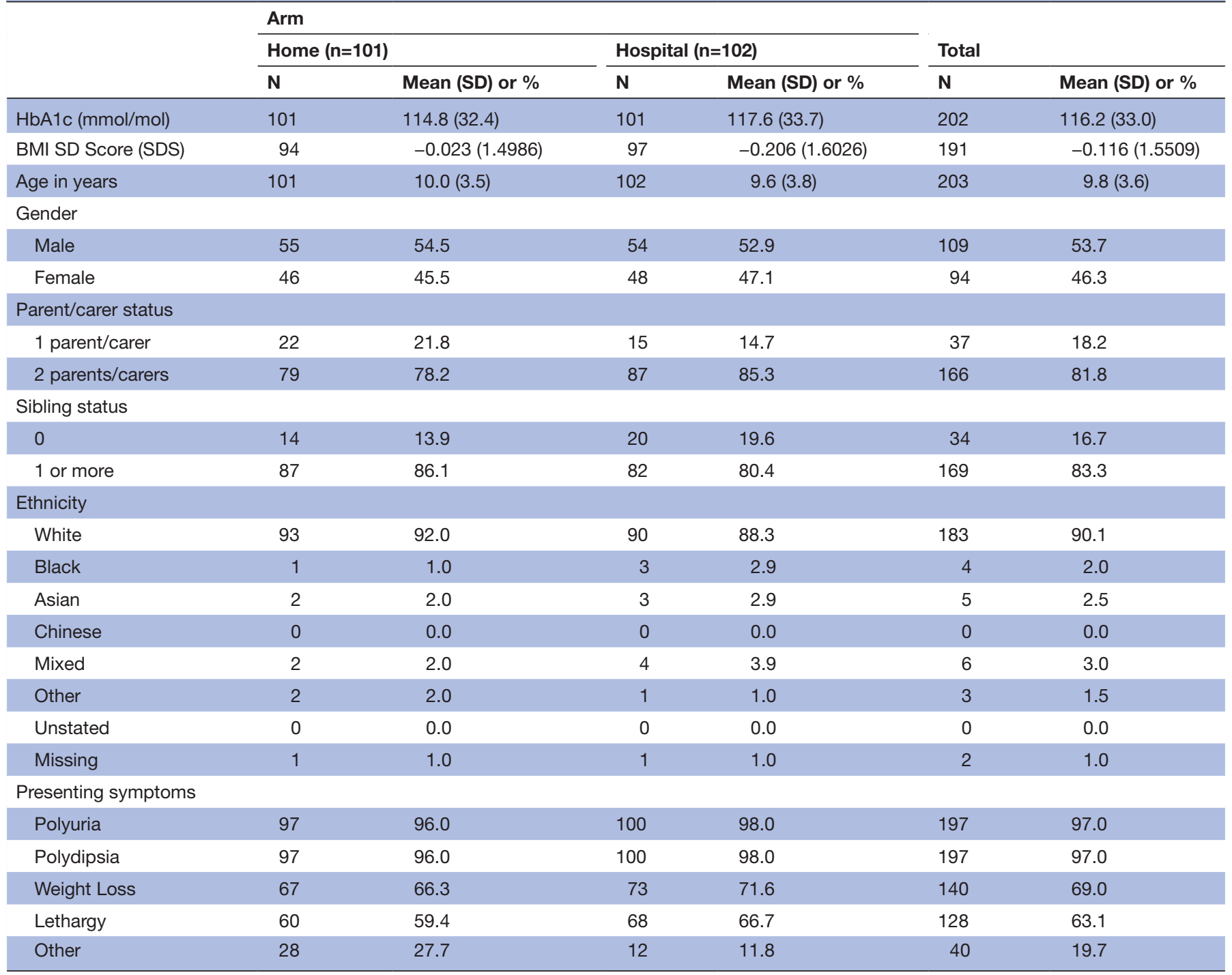

BMI, body mass index; HbA1c, glycosylated haemoglobin.

A coexisting chronic disorder (eg, cystic fibrosis) that would impact independently but significantly on blood glucose control.

- A diagnosis of type 2 diabetes or maturity onset diabetes of the young.
- An uncertain diagnosis.

- A sibling with T1D.

- Children who were to begin treatment on a continuous subcutaneous insulin infusion.

- Children under the care of the local authority.

Table 2 Primary outcome-HbA1c at 24 months by arm controlling for $\mathrm{HbA} 1 \mathrm{c}$ at baseline

\begin{tabular}{|c|c|c|c|c|c|c|c|c|c|c|c|c|c|}
\hline \multirow[b]{3}{*}{ Outcome } & \multicolumn{5}{|c|}{ Home } & \multicolumn{5}{|c|}{ Hospital } & \multirow{3}{*}{$\begin{array}{l}\text { Difference } \\
\text { in means }\end{array}$} & \multirow[b]{3}{*}{$95 \% \mathrm{Cl}$} & \multirow[b]{3}{*}{$P$ value } \\
\hline & \multirow[b]{2}{*}{$\mathbf{N}$} & \multicolumn{2}{|c|}{ Baseline } & \multicolumn{2}{|c|}{24 months } & \multirow[b]{2}{*}{$\mathbf{N}$} & \multicolumn{2}{|c|}{ Baseline } & \multicolumn{2}{|c|}{24 months } & & & \\
\hline & & Mean & SD & Mean & SD & & Mean & SD & Mean & SD & & & \\
\hline $\begin{array}{l}\mathrm{HbA1c}(\mathrm{mmol} / \\
\mathrm{mol}) \dagger\end{array}$ & 100 & 114.3 & 32.1 & 71.9 & 21.56 & 98 & 116.9 & 33.5 & 72.8 & 22.0 & 1.01 & 0.93 to 1.09 & 0.858 \\
\hline
\end{tabular}

Model covariate is natural log transformation of Baseline $\mathrm{HbA} 1 \mathrm{c}$. Reference category is Hospital. Effect/95\% $\mathrm{Cl}$ are back-transformed from a natural log transformation and hence are on the ratio level.

*Of the 96 patients in the Hospital arm with HbA1c recorded at 24 months, baseline HbA1c was missing for one.

†This result is a sensitivity analysis including replaced $\mathrm{HbA1c}$ values.

HbA1c, glycosylated haemoglobin. 


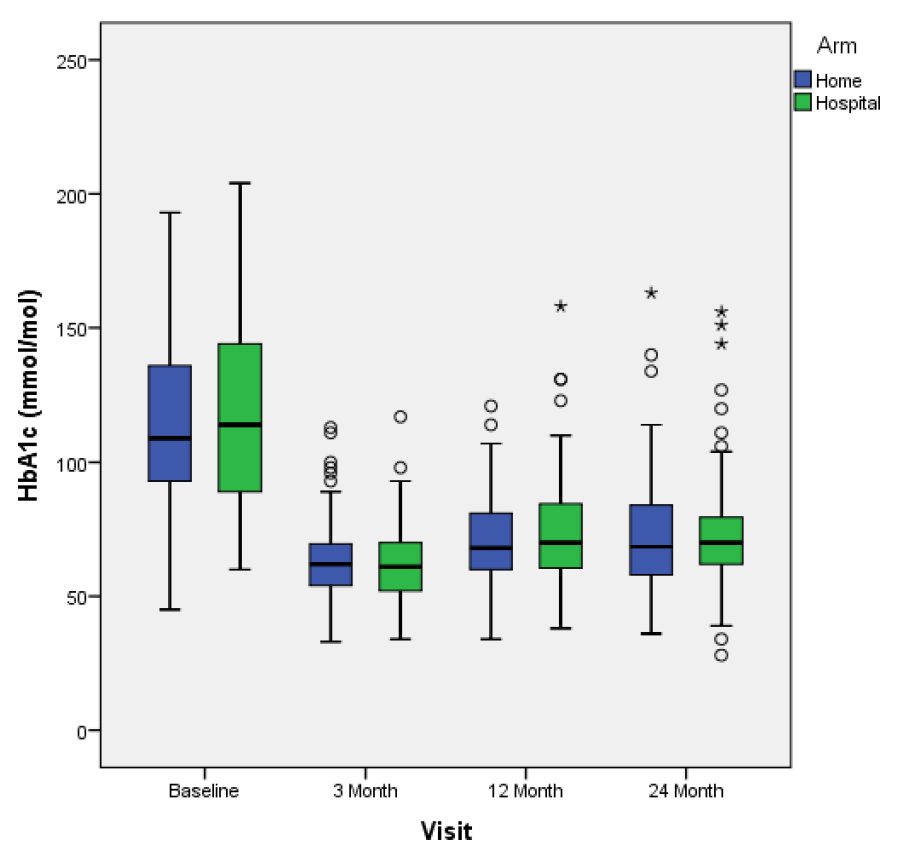

Figure 2 Median and IQRs of HbA1c at each follow-up visit. $\mathrm{HbA1c}$, glycosylated haemoglobin.

- Children whose home circumstances were deemed unsuitable for home management.

Sample size and recruitment: To have $80 \%$ power to detect an effect size of 0.4 (difference in mean HbAlc of $5 \mathrm{mmol} / \mathrm{mol}$ with an SD of $14 \mathrm{mmol} / \mathrm{mol}^{6}$ ) at a $5 \%$ significance level, 200 participants were required. To allow for loss to follow-up of 17\%, a target of 240 participants was set for recruitment. A previous study in Canada which evaluated reducing the amount of inpatient time at diagnosis showed a difference of $7.65 \mathrm{mmol} / \mathrm{mol}$ in mean HbAlc at 2 years. ${ }^{9}$ Within each centre, a trialspecific DECIDE research nurse was employed to co-ordinate nursing management of participants and data collection. Participants were recruited by paediatric diabetes team members or DECIDE research nurses. Participants (aged over 8 years) and all parents/carers of participants (aged $<17$ years) were given information about the trial to read while in the assessment unit/ paediatric ward. They had time to consider participating in the trial while blood tests were taken to confirm the clinical diagnosis.

\section{Randomisation and masking}

Once informed consent/assent was obtained, participants were randomised to hospital management or home management using an automated telephone system operational 24 hours a day. Randomisation was computer generated and stratified by centre and balanced using randomly chosen permuted blocks. The randomisation ratio was 1:1. It was not possible to blind participants and those delivering the intervention. However, the blood samples collected for the primary outcome of HbA1c were analysed blind to study group.
Intervention

Home management

Home management was defined as involving no overnight stay in hospital. Standard elements of home management common to all participating centres were:

- Discharge home on the day of diagnosis with no overnight admission to hospital.

- All subsequent treatment, face to face education about diabetes and its management by nursing staff and emotional/practical support was to be delivered at home or on an outpatient basis. Outpatient visits were defined as attending ward/clinic for no longer than 2 hours for supervision of injections as necessary according to local need, for a minimum of 3 days (the initiation period); this time frame provided uniformity across the two trial arms.

- Dietetic education at home or as an outpatient.

- Early team review to reflect on the team's experience of home-based care and to address identified problems.

\section{Hospital management}

Children randomised to the hospital arm were admitted at diagnosis, with an intended minimum stay of three nights during the initiation period. During their inpatient stay, families received treatment and education similar in nature to that received by those in the home-based arm and multidisciplinary support in the ward environment consistent with local practice.

\section{Home and hospital management}

Each participating centre was provided with one research grant-funded DECIDE Study nurse for the duration of the trial. They were trained, where needed, to provide diabetes care by the local diabetes teams, as well as having access to support from the lead centre (Cardiff), as required. How the DECIDE research nurse was integrated into the team varied between centres for logistical reasons. In some centres, provision of education and support to newly diagnosed participants and their families in both study arms was shared equally with other paediatric diabetes team members, whereas in others, the DECIDE research nurses undertook all the home management.

As a pragmatic, randomised controlled trial allowing for different approaches to providing clinical care, participating centres were not given specific, detailed instructions of what should be delivered in terms of diabetes education and support. Families in both arms received written information about diabetes and diabetes education as routinely delivered by individual centres. Centres were asked to provide the same education programme to the newly diagnosed and their families, regardless of location of initial care and no attempt was made to influence their normal approaches. Children were advised on personalised insulin dose changes dependent on the results of blood glucose testing, with the aim of optimising glycaemic control as soon as possible after diagnosis. To facilitate delivery of the trial, a DECIDE manual 


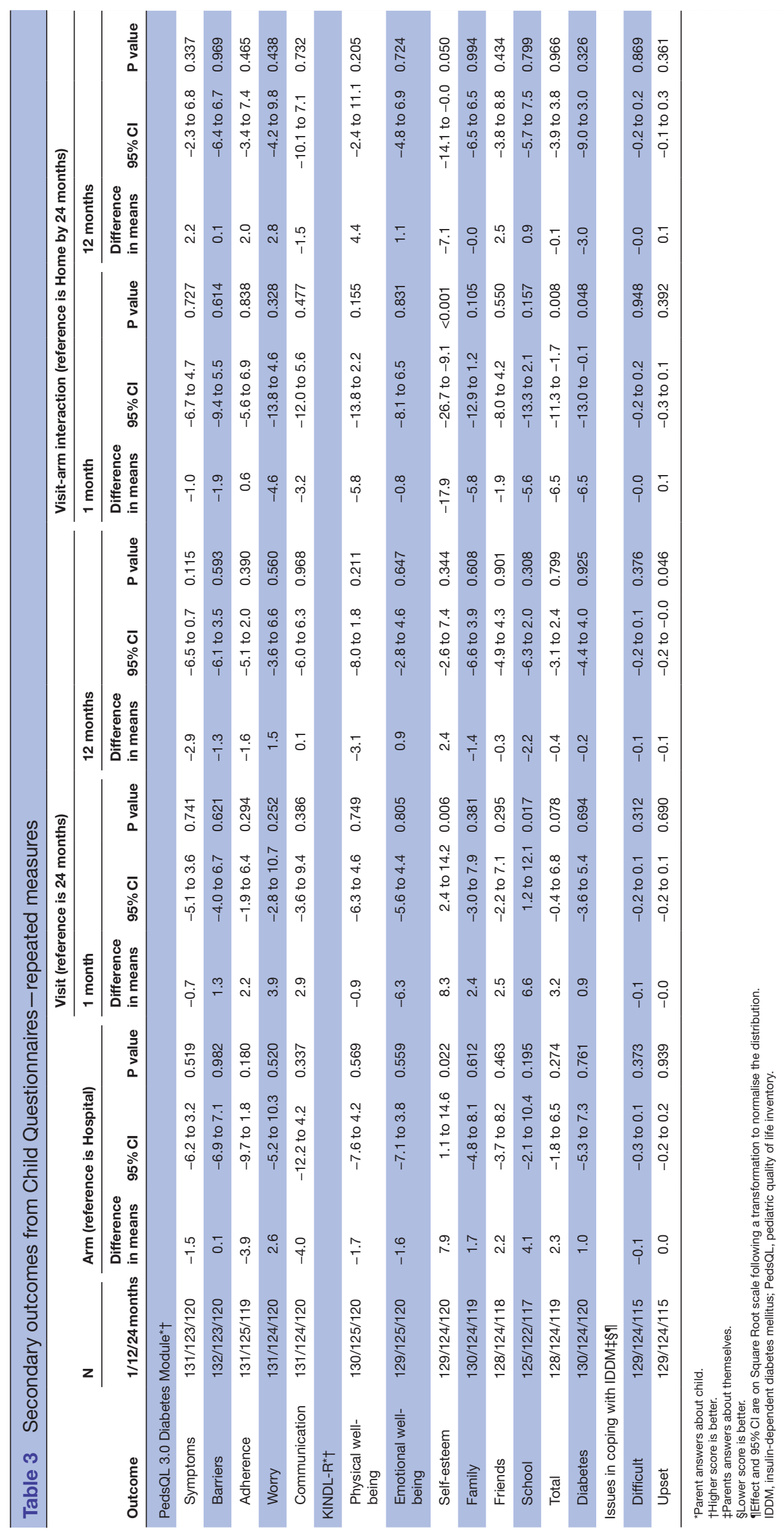


Table 4 Secondary outcomes from Child Questionnaires-comparison at 1 month

\begin{tabular}{|c|c|c|c|c|c|c|c|c|c|}
\hline \multirow[b]{3}{*}{ Outcome } & \multicolumn{3}{|c|}{ Home } & \multicolumn{3}{|c|}{ Hospital } & \multirow{3}{*}{$\begin{array}{l}\text { Difference } \\
\text { in means }\end{array}$} & \multirow[b]{3}{*}{$95 \% \mathrm{Cl}$} & \multirow[b]{3}{*}{$P$ value } \\
\hline & \multirow[b]{2}{*}{$\mathbf{N}$} & \multicolumn{2}{|c|}{1 month } & \multirow[b]{2}{*}{$\mathbf{N}$} & \multicolumn{2}{|c|}{1 month } & & & \\
\hline & & Mean & SD & & Mean & SD & & & \\
\hline Symptoms & 69 & 60.2 & 14.23 & 62 & 62.3 & 13.09 & -2.1 & -6.8 to 2.6 & 0.384 \\
\hline Worry & 68 & 72.7 & 24.26 & 63 & 74.7 & 22.94 & -2.1 & -10.2 to 6.1 & 0.616 \\
\hline Communication & 68 & 72.9 & 28.01 & 63 & 81.3 & 18.25 & -8.4 & -16.7 to -0.2 & 0.045 \\
\hline \multicolumn{10}{|l|}{ KINDL-R ${ }^{\star} \dagger$} \\
\hline Physical well-being & 68 & 63.0 & 20.38 & 62 & 70.4 & 19.07 & -7.5 & -14.3 to -0.6 & 0.033 \\
\hline Friends & 68 & 79.3 & 14.62 & 60 & 78.6 & 16.33 & 0.5 & -4.8 to 5.9 & 0.849 \\
\hline School & 65 & 67.0 & 21.92 & 60 & 68.1 & 18.65 & -1.1 & -8.3 to 6.1 & 0.763 \\
\hline Total & 67 & 69.2 & 13.78 & 61 & 73.2 & 12.45 & -3.9 & -8.6 to 0.7 & 0.092 \\
\hline Diabetes & 68 & 72.2 & 19.16 & 62 & 77.9 & 15.76 & -5.7 & -11.7 to 0.4 & 0.068 \\
\hline
\end{tabular}

Issues in coping with

IDDM $\ddagger \S 9$

$\begin{array}{llllllllll}\text { Difficult } & 67 & 17.6 & 3.53 & 62 & 16.7 & 3.20 & -0.1 & -0.2 \text { to } 0.0 & 0.148 \\ \text { Upset } & 67 & 16.5 & 4.70 & 62 & 15.6 & 3.96 & -0.1 & -0.3 \text { to } 0.1 & 0.271\end{array}$

Reference category is Hospital.

*Parent answers about Child.

†Higher score is better.

$\ddagger$ Parents answers about themselves.

$\S$ Lower score is better.

ПEffect and $95 \% \mathrm{Cl}$ are on Square Root scale following a transformation to normalise the distribution.

IDDM, insulin-dependent diabetes mellitus; PedsQL, pediatric quality of life inventory.

was provided to all centres, offering guidance in key areas such as initial diagnosis, recruitment, home management and hospital management. The manual recommended:

- That the insulin regime should be determined by local practice but suggested a starting dose of approximately $0.5 \mathrm{U} / \mathrm{kg} /$ day adjusted flexibly in light of individual response.

- A minimum of four capillary glucose checks daily and a target range of blood glucose concentrations of 4-9 $\mathrm{mmol} / \mathrm{L}$.

All families were given an appointment to attend the next appropriate diabetes clinic, received continued support from health professionals through telephone contact and clinic visits and were able to access help and advice out of office hours. Thereafter, participants randomised to both arms received outpatient appointments in line with local practice and individual need.

\section{Outcomes}

The primary outcome was children's glycaemic control (HbAlc analysed at a central laboratory) 24 months postdiagnosis when most participants would have no significant endogenous insulin secretion, thus providing an effective measure of diabetes management.

Secondary outcomes concerning participants were $\mathrm{HbAlc}$ at 3 and 12 months postdiagnosis (analysed at a central laboratory) and questionnaire-based psychological assessment of quality of life $\left((\text { KINDL-R })^{17}\right.$ and PedsQL 3.0 Diabetes Module, ${ }^{18}$ issues in coping with $\mathrm{T}_{1} \mathrm{D}^{19}$ and diabetes knowledge ${ }^{20}$ at 1,12 and 24 months. Secondary outcomes concerning parent(s)/carers were anxiety, ${ }^{21}$ issues in coping with $\mathrm{T} 1 \mathrm{D},{ }^{22}$ quality of life $\left((\text { KINDL-R })^{17}\right.$ and PedsQL 3.0 Diabetes Module ${ }^{18}$ ) and diabetes knowledge ${ }^{20}$ at 1,12 and 24 months. The impacts on social activities and independence were measured using a bespoke questionnaire developed for this trial, which will be reported separately. All questionnaires were self-completed where possible in those aged 8 years and over at trial entry to ensure independent answers from children and their parents. To monitor safety, numbers of serious adverse events (SAEs) per 
participant were collected. Growth (height, weight and body mass index) was measured at baseline, 3, 12 and 24 months. NHS resource use for the initiation period (days $0-3)$ and for the follow-up period to 24 months, and days off work and school in the initiation period were also documented.

\section{Resource use}

The intervention did not require additional training for existing healthcare teams over and above the DECIDE manual for guidance about home-based and hospitalbased care for those not previously experienced in providing either model of care. Data on NHS resource use were collected, which covered initiation plus the full period of follow-up at 3,12 and 24 months.

\section{Statistical analysis}

The primary analysis followed intention to treat principles for the complete case data set. Comparison of mean HbAlc between the two arms at the 24 month follow-up time point was carried out including baseline HbAlc as a covariate. HbAlc data were transformed to normality using a natural log transformation. The difference between arms in HbAlc, the intervention effect, is therefore interpreted as percentage difference. This analysis was corrected for any clustering of outcomes within centres by using a mixed 2-level hierarchical linear model.

Repeated measures secondary analysis of the primary outcome using a generalised linear model was carried out using the 3,12 and 24 month data with baseline data as a covariate. This and the primary analysis were repeated as sensitivity analyses with missing laboratory data replaced by available routine trial site HbAlc measurements taken at the relevant timepoints. Where laboratory HbAlc data were reported in percentages, data were converted to $\mathrm{mmol} / \mathrm{mol}$ using the equation $[\mathrm{mmol} / \mathrm{mol}]=10.93 *(\%)$ $-23.5 .^{23}$

Secondary questionnaire outcomes analyses (except diabetes knowledge) compared the two arms using a mixed model at 1 and 24 months and assessed repeated measures across 1, 12 and 24 months through a generalised linear model. These analyses also corrected for any clustering of outcomes within centre by using 2-level hierarchical versions of these models. Diabetes knowledge and days off work and school were compared using the Mann-Whitney test. Secondary growth outcomes analyses compared the two arms using a mixed model at 24 months and assessed repeated measures across 1, 12 and 24 months through a generalised linear model, all with Baseline data as a covariate. These analyses also corrected for any clustering of outcomes within centre by using 2-level hierarchical versions of these models.

$P$ values are to be interpreted with caution given the number of statistical tests undertaken here $(n=213)$. Only $\mathrm{p}<0.001$ can be considered statistically significant for all following analyses.

\section{Qualitative analysis}

The burden of the trial and a process evaluation was assessed through qualitative interviews of young people and their families ${ }^{24}$ and healthcare professional participants (awaiting publication).

\section{Trial governance}

Multicentre approval was granted by Research Ethics Committee for Wales (07/MRE09/59). Site-specific approval was granted by participating Acute Trust Research and Development Departments. The trial sponsor was Cardiff University.

\section{Patient and public involvement}

Two parents of children diagnosed with T1D were involved in the initial design of the DECIDE trial. One of these parents was a coapplicant on the funding application and was instrumental in ensuring that the trial was informed by the families' experience. She also attended the ethics committee meeting to provide a service user perspective of the value of the trial to inform the committee's decision. She and another parent were part of the Trial Management Group which met monthly and provided input on the conduct of the trial throughout.

A dissemination day for the public and clinical staff from all the centres was arranged at the end of the trial, at which preliminary results were presented and their implications discussed with lay participants. A newsletter summarising results was also sent to all participants for their information. Discussions from the dissemination day helped to shape this paper.

\section{RESULTS}

Two hundred and three children aged $<17$ years newly diagnosed with T1D and their parents/carers were recruited over 40 months from July 2008 to October 2011 to either hospital $(n=102)$ or home management $(n=101)$ (figure 1). There were reasonable similarities across the two arms on important baseline characteristics including age, gender and the number of parents/carers (table 1).

There was no difference $(\mathrm{p}=0.863)$ in the primary outcome of mean HbA1c at24 months (controlling for $\mathrm{HbA1c}$ at baseline and clustering within centres) between initial management at home $(72.1 \mathrm{mmol} / \mathrm{mol})$ and hospital (72.6 $\mathrm{mmol} / \mathrm{mol})$ (table 2$)$.

This was unchanged in the secondary repeated measures analysis and in sensitivity analyses (in which 14 of the 22 missing HbAlc values across all time points were replaced with trial site measurements), with no differences between arms seen. Both repeated measures analyses indicated that participants had significantly lower ( $<<0.001)$ HbA1c at 3 months (home: mean $(63.7 \mathrm{mmol} /$ mol), median $(62.0 \mathrm{mmol} / \mathrm{mol})$; hospital: mean $(62.5 \mathrm{mmol} / \mathrm{mol})$, median $(61.0 \mathrm{mmol} / \mathrm{mol})$ compared with 24 months (figure 2).

While the primary analysis focused on the final 24-month time point, the secondary outcomes were 
Table 5 Secondary outcomes from Child Questionnaires-comparison at 24 months

\begin{tabular}{|c|c|c|c|c|c|c|c|c|c|}
\hline \multirow[b]{3}{*}{ Outcome } & \multicolumn{3}{|c|}{ Home } & \multicolumn{3}{|c|}{ Hospital } & \multirow{3}{*}{$\begin{array}{l}\text { Difference } \\
\text { in means }\end{array}$} & \multirow[b]{3}{*}{$95 \% \mathrm{Cl}$} & \multirow[b]{3}{*}{$P$ value } \\
\hline & \multirow[b]{2}{*}{$\mathbf{N}$} & \multicolumn{2}{|c|}{24 months } & \multirow[b]{2}{*}{$\mathbf{N}$} & \multicolumn{2}{|c|}{24 months } & & & \\
\hline & & Mean & SD & & Mean & SD & & & \\
\hline Symptoms & 62 & 62.0 & 12.56 & 58 & 63.3 & 14.11 & -1.2 & -5.9 to 3.6 & 0.633 \\
\hline Barriers & 62 & 74.4 & 19.21 & 58 & 74.2 & 20.75 & 0.1 & -7.1 to 7.3 & 0.975 \\
\hline Worry & 62 & 73.3 & 20.75 & 58 & 71.1 & 23.74 & 2.1 & -5.9 to 10.2 & 0.601 \\
\hline Communication & 62 & 72.8 & 25.83 & 58 & 78.2 & 21.22 & -5.5 & -14.0 to 3.0 & 0.200 \\
\hline \multicolumn{10}{|l|}{ KINDL-R* } \\
\hline Physical well-being & 62 & 70.0 & 17.64 & 58 & 71.0 & 15.90 & -1.0 & -7.1 to 5.1 & 0.741 \\
\hline Emotional well-being & 62 & 76.6 & 18.18 & 58 & 78.6 & 12.35 & -2.0 & -7.7 to 3.6 & 0.482 \\
\hline School & 60 & 65.9 & 17.32 & 57 & 61.5 & 18.14 & 4.6 & -1.9 to 11.0 & 0.163 \\
\hline Total & 61 & 72.6 & 12.77 & 58 & 70.4 & 11.42 & 2.3 & -2.1 to 6.7 & 0.308 \\
\hline Diabetes & 62 & 76.8 & 18.55 & 58 & 76.5 & 18.07 & 0.3 & -6.3 to 6.9 & 0.931 \\
\hline \multicolumn{10}{|c|}{ Issues in coping with IDDM†‡ } \\
\hline Difficult & 59 & 17.2 & 4.94 & 56 & 16.3 & 4.44 & -0.1 & -0.3 to 0.1 & 0.199 \\
\hline Upset & 59 & 15.5 & 5.03 & 56 & 15.6 & 4.54 & 0.0 & -0.2 to 0.2 & 0.897 \\
\hline
\end{tabular}

Reference category is Hospital.

*Higher score is better.

†Lower score is better.

†Effect and $95 \% \mathrm{Cl}$ are on Square Root scale following a transformation to normalise the distribution.

IDDM, insulin-dependent diabetes mellitus; PedsQL, pediatric quality of life inventory.

collected at 1-month and 12-month interim time points. Hence, repeated measures analysis have been performed and presented in full in tables 3-5. Here, the main effects of trial arm are given along with the effect at the interim time periods. The final columns provide the interaction of arm with time and significant effects here indicate a differential effect of the intervention over time between arms. For children, there was one differential effect over time between trial arms which was self-esteem at 1 month $(\mathrm{p}<0.001)$ (table 3). The children in the hospital arm had higher self-esteem than those in the home arm at the 1 month time point, with the opposite being the case at the 24 month time point.

There were no differences between trial arms in secondary outcomes for parents at 1 month (table 6), nor at 24 months (table 7), which is mirrored in the repeated measures analyses (table 8).

For growth outcomes, there were no differences between arms at 24 months or when viewed at repeated measures across 3, 12 and 24 months. For height and weight, measurements at 3 and 12 months were significantly less than those at 24 months, with no evidence of excess weight gain in either arm (online supplementary tables 7,8$)$.
The initiation period for this study was the first 3 days. Since in the hospital arm the patients were already admitted, no SAEs relating to hospitalisation could be recorded. No children in the home arm were admitted to hospital from the home arm during the initiation period and there were no adverse events at that time. SAE data were also collected during the 2-year follow-up in both arms and more SAEs were reported in participants managed in the home arm (online supplementary table 9). $95 \%$ CIs for the difference in proportion of patients reporting SAEs and total events reported are also given in the table and none indicate significant differences in SAE rates between arms. For further information the SAEs were categorised as diabetes associated or not, most SAE events were hypoglycaemic or hyperglycaemic events (online supplementary table 10).

NHS resource uses costing data source and unit cost is shown in online supplementary table 11 with median use of NHS resources shown in online supplementary table 12. Almost all participants in the hospital management group spent the prescribed three nights in hospital (online supplementary table 13). No participants in the home management group were admitted to hospital during the initiation period. There were fewer contacts 
Table 6 Secondary outcomes from Parent Questionnaires - comparison at 1 month

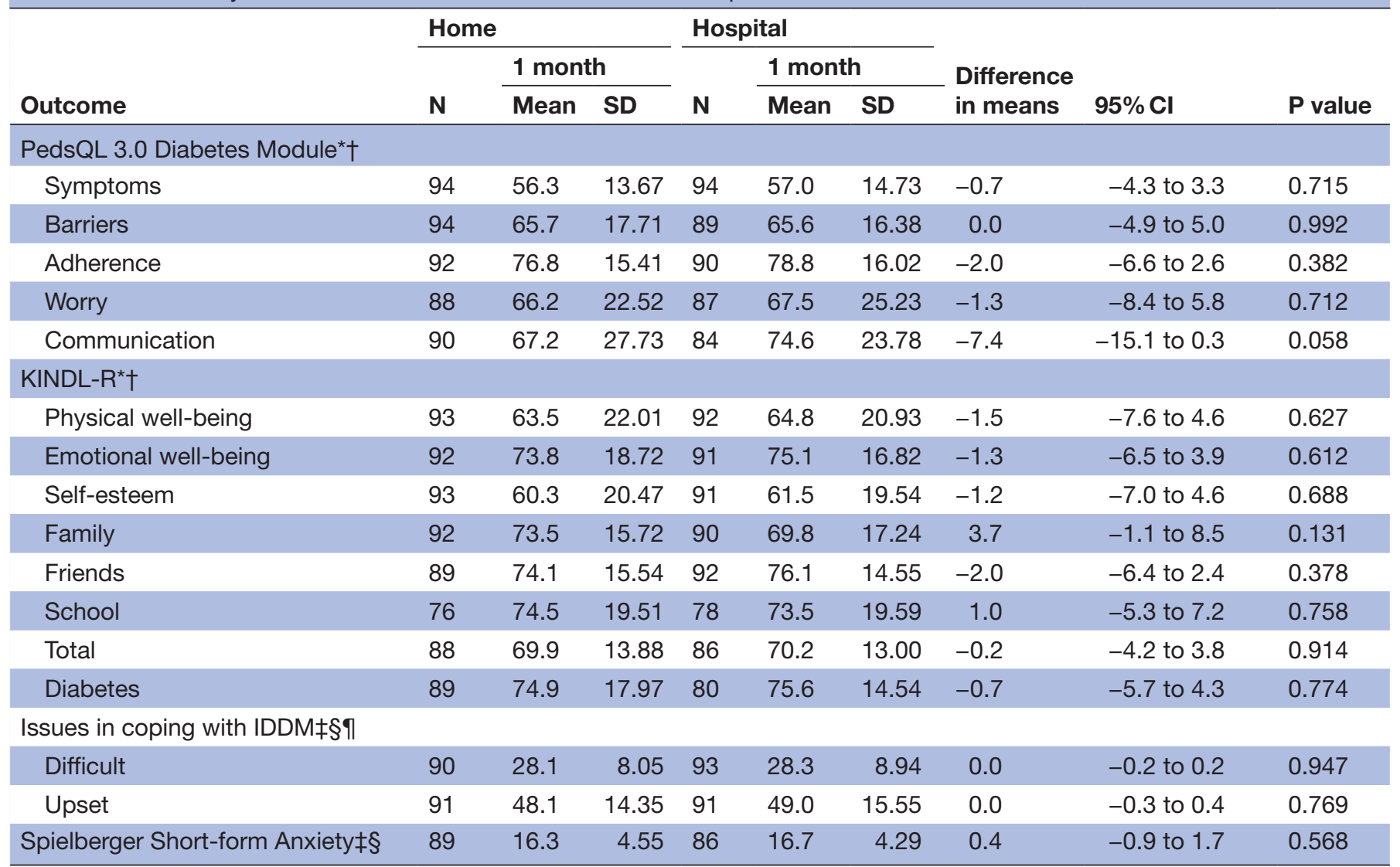

Reference category is Hospital.

*Parent answers about Child.

†Higher score is better.

$\ddagger$ Parents answers about themselves.

$\S$ Lower score is better.

IEffect and $95 \% \mathrm{Cl}$ are on Square Root scale following a transformation to normalise the distribution.

IDDM, insulin-dependent diabetes mellitus; PedsQL, pediatric quality of life inventory.

(online supplementary table 12) with consultants (median 1 vs 2) and junior doctors (median 1 vs 3 ) in the home management group. Face to face contacts with diabetes nurses were similar between groups (median 6 for both groups) but non face-to-face contacts were higher for the home management group (median 2 vs 0 ), as were miles travelled by the diabetes nurses (median 40.3 vs 0.0 miles). Contacts with dietitians were similar between groups. During the follow-up period, resource use was similar between groups. There was no difference between trial arms during the initiation period in the time that parents needed to take off work or the child off school (online supplementary table 14).

\section{DISCUSSION}

This multicentre individually randomised trial demonstrates that clinical and educational care for newly diagnosed children with T1D whether delivered at home or in hospital has no effect on blood glucose control, psychological outcomes or SAEs. Both models of care seem acceptable to young people and their families. ${ }^{24}$
There was a change in the balance of who delivered care with a greater focus on nurse delivery in the home arm than in hospital, where care was shared more between professional groupings. These findings are important as they provide an evidence base for clinical practice that to date has been largely informed by clinical anecdote. Our findings challenge established clinical practice as well as inform those responsible for the provision of health services who wish to redesign care pathways with more care provided in the community.

This is the largest randomised trial in any country examining the effect of location of clinical and educational care at diagnosis for children with T1D. By including both tertiary and secondary care hospitals and a representative sample of young people with newly diagnosed T1D, we believe that the findings of this trial may be considered generalisable across a range of healthcare systems. The prospective nature of this trial and randomisation within centres ensured that potential recruitment bias was kept to a minimum. The trial had high follow-up rates and therefore will have unbiased estimates of treatment 
Table 7 Secondary outcomes from Parent Questionnaires-comparison at 24 months

\begin{tabular}{|c|c|c|c|c|c|c|c|c|c|}
\hline \multirow[b]{3}{*}{ Outcome } & \multicolumn{3}{|c|}{ Home } & \multicolumn{3}{|c|}{ Hospital } & \multirow{3}{*}{$\begin{array}{l}\text { Difference } \\
\text { in means }\end{array}$} & \multirow[b]{3}{*}{$95 \% \mathrm{Cl}$} & \multirow[b]{3}{*}{$P$ value } \\
\hline & \multirow[b]{2}{*}{$\mathbf{N}$} & \multicolumn{2}{|c|}{24 months } & \multirow[b]{2}{*}{$\mathbf{N}$} & \multicolumn{2}{|c|}{24 months } & & & \\
\hline & & Mean & SD & & Mean & SD & & & \\
\hline Symptoms & 85 & 59.1 & 12.80 & 87 & 57.2 & 14.45 & 2.0 & -2.1 to 6.1 & 0.708 \\
\hline Barriers & 85 & 67.3 & 19.11 & 86 & 63.7 & 20.82 & 3.6 & -2.4 to 9.7 & 0.238 \\
\hline Worry & 83 & 70.2 & 20.58 & 85 & 70.6 & 22.00 & -0.4 & -6.9 to 6.1 & 0.901 \\
\hline Communication & 79 & 69.7 & 26.26 & 82 & 73.3 & 21.82 & -3.5 & -11.1 to 4.0 & 0.352 \\
\hline \multicolumn{10}{|l|}{ KINDL-R* ${ }^{\star}$} \\
\hline Physical well-being & 85 & 70.3 & 17.81 & 86 & 65.9 & 19.07 & 4.4 & -1.2 to 9.9 & 0.123 \\
\hline Emotional well-being & 84 & 76.2 & 16.12 & 87 & 76.3 & 14.58 & -0.1 & -4.7 to 4.6 & 0.982 \\
\hline Friends & 83 & 77.0 & 15.12 & 88 & 75.6 & 14.89 & 1.4 & -3.1 to 5.9 & 0.545 \\
\hline School & 81 & 75.2 & 17.25 & 80 & 72.1 & 18.1 & 3.1 & -2.4 to 8.6 & 0.270 \\
\hline Total & 83 & 72.1 & 11.27 & 87 & 70.1 & 11.86 & 2.1 & -1.4 to 5.6 & 0.244 \\
\hline Diabetes & 84 & 77.9 & 14.95 & 86 & 75.8 & 15.66 & 2.1 & -2.5 to 6.8 & 0.363 \\
\hline \multicolumn{10}{|c|}{ Issues in coping with IDDM $\neq \S \rrbracket$} \\
\hline Difficult & 83 & 25.9 & 8.79 & 88 & 26.8 & 9.20 & 0.1 & -0.2 to 0.3 & 0.539 \\
\hline Upset & 84 & 45.5 & 17.10 & 87 & 47.9 & 17.53 & 0.2 & -0.2 to 0.5 & 0.358 \\
\hline $\begin{array}{l}\text { Spielberger Short-form } \\
\text { Anxietyt§ }\end{array}$ & 84 & 14.8 & 4.65 & 81 & 15.1 & 4.95 & -0.3 & -1.8 to 1.2 & 0.701 \\
\hline
\end{tabular}

Reference category is Hospital.

*Parent answers about Child.

†Higher score is better.

$\ddagger$ Parents answers about themselves.

§Lower score is better.

qEffect and $95 \% \mathrm{Cl}$ are on Square Root scale following a transformation to normalise the distribution.

IDDM, insulin-dependent diabetes mellitus; PedsQL, pediatric quality of life inventory.

effects. We also used a central laboratory for HbA1c assays thereby reducing between site variation in results. ${ }^{25}$

As most participating centres had no prior experience of home care from diagnosis, careful consideration was given to defining and manualising key components of home and hospital care, to provide inexperienced centres with adequate guidelines and to ensure reproducibility of the intervention. This intervention was informed by a comprehensive multidisciplinary research team with input from experts in paediatric diabetes medicine, nursing, psychology and complex trials as well as parents of children with T1D and was deemed acceptable by young people and families. ${ }^{24}$

\section{Limitations}

Research-funded nurses were employed in the participating centres. This extra resource, alongside uncertainties about its impact, may limit the extrapolation from the findings of this trial to routine clinical practice as it is possible that home-care may prove unfeasible without additional specialist nursing resources. It may be that a hybrid model, of a brief stay in hospital and early home management, offers a pragmatic solution to the challenges presented by both systems as has been tested in Canada, Sweden and Australia. ${ }^{9} 2627$

Methodological limitations of our trial included the unblindable nature of the intervention and the potential risk of practice in one arm influencing delivery of the other arm. Also, we recruited 203 subjects rather than the 240 originally intended which was to allow for drop-out and a final sample of 200 to give the trial adequate power. However, through a high rate of follow-up, only one subject dropped out and 194 individuals provided HbAlc samples to measure our primary outcome. It is unlikely that obtaining samples from six additional subjects would have altered our statistical findings.

\section{Context within current literature}

In 2007, a Cochrane Systematic Review ${ }^{6}$ concluded there were insufficient data to determine whether home or outpatient management of children newly diagnosed with T1D but not acutely unwell was as good as, or better than, 


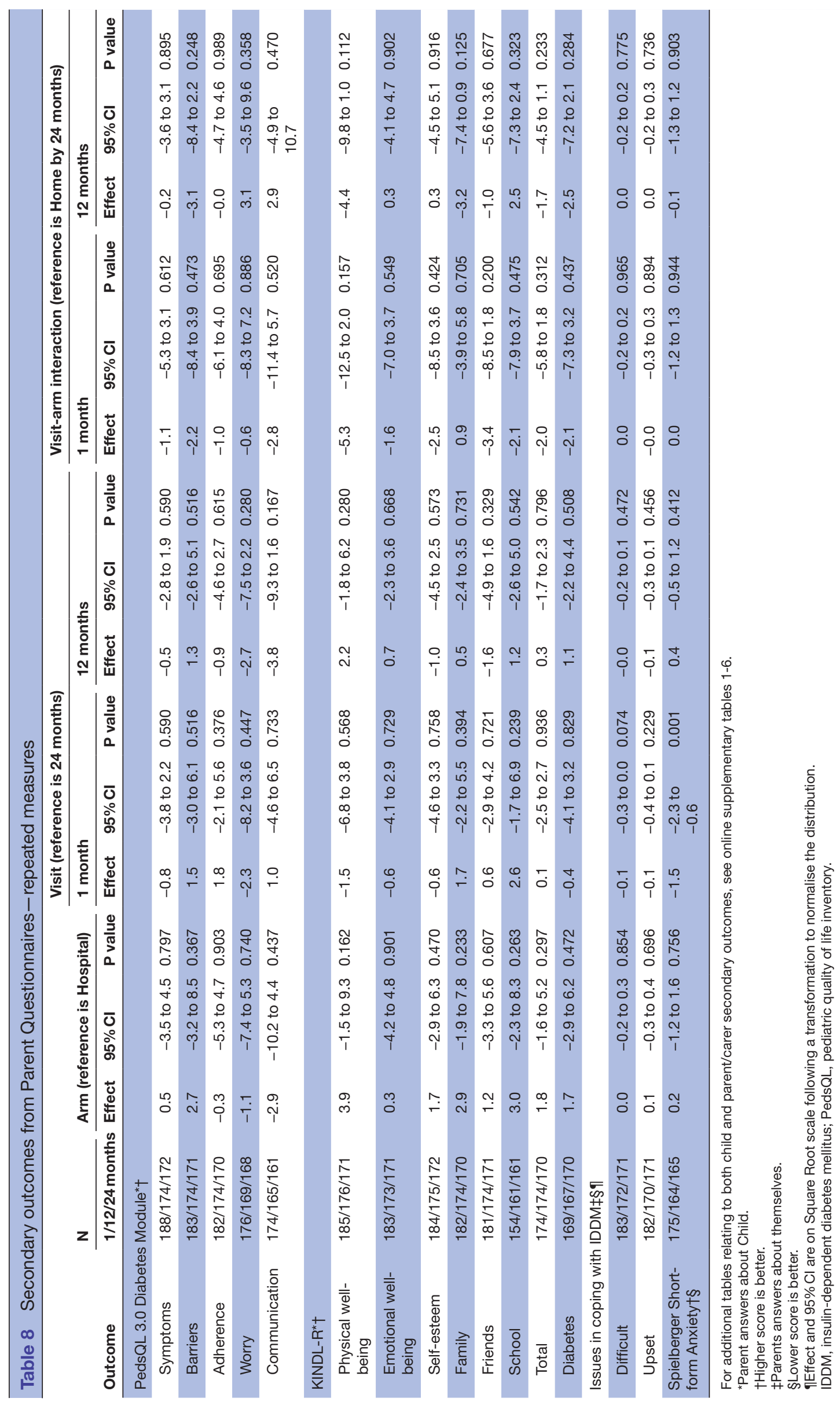


inpatient care. Worldwide, we have identified only four randomised controlled trials, in Finland, Canada, Sweden and Australia, all of which tested hybrid models, involving short periods (2-9 days) of inpatient care. None of these trials have shown any clinically important differences in outcomes. $^{9} 15$ 26-28

DECIDE is the only multicentre, randomised controlled trial worldwide that has achieved an adequate sample to provide statistical power. An important difference from previously published studies ${ }^{91526-28}$ is that no participants in the home arm of this trial were admitted to hospital overnight at diagnosis or were admitted to hospital during initiation of treatment. Our finding of no difference in longer term metabolic outcome or major quality of life measures is consistent with the findings of these other trials as well as recent non-randomised studies and suggests that even relatively early discharge does not produce adverse outcomes across a range of services in a UK setting. ${ }^{29}$ Furthermore, the similarity of findings across countries with differing models of healthcare and diabetes metabolic outcomes suggest that where care is delivered after diagnosis is less important than the quantity and quality of the support provided to families.

\section{Implications for clinical practice}

In England, changes have occurred since completion of this trial in the commissioning and delivery of services for children with diabetes, with a greater focus on education and a more aggressive approach to insulin therapy ${ }^{30}$ at diagnosis than has traditionally been the case. ${ }^{31} 32$ This means that if the principles of this trial were to be rolled out in clinical practice in a UK-setting, some updating of the home-based intervention would be required. This would need to include analyses to identify the key components of these more intensive packages of care, to determine how they could be realistically incorporated into a home-based model of care in a safe, economic and clinically effective way, given that prolonged hospitalisation for several weeks education in a UK setting is probably unaffordable. Hybrid models of care as evaluated in Sweden, Canada and Western Australia ${ }^{9} 26$ that include a short initial period of hospitalisation may prove necessary to incorporate ideal principles from both models of care.

\section{CONCLUSION}

The findings of this trial suggest that there is no evidence of a difference between home-based and hospital-based initiation of care to children newly diagnosed with T1D across relevant outcomes. Given that hospitalisation at diagnosis is costly for the NHS, consideration should be given as to how the currently more intensive packages of care that are offered to the newly diagnosed might be provided in the context of early discharge to home-based care.

\section{Author affiliations}

${ }^{1}$ Cardiff University, School of Medicine, Cardiff, UK

${ }^{2}$ Centre for Trials Research, Cardiff University, Cardiff, UK
${ }^{3}$ Health Economics and Policy Research Unit, University of South Wales, Pontypridd, UK

${ }^{4}$ Marie Curie Palliative Care Research Centre, Cardiff University, School of Medicine, Cardiff, UK

${ }^{5}$ Southampton Children's Hospital, University Hospital Southampton NHS Foundation Trust, Southampton, UK

${ }^{6}$ University of Liverpool, Institute of Translational Medicine, Liverpool, UK ${ }^{7}$ Nottingham Children's Hospital, Nottingham University Hospitals NHS Trust, Nottingham, UK

${ }^{8}$ School of Biosciences and Medicine, University of Surrey, Guildford, UK ${ }^{9}$ Department of Child Health, Cardiff and Vale University Health Board, Cardiff, UK ${ }^{10}$ School of Healthcare Sciences, Cardiff University, Cardiff, UK

\section{Twitter Morro Touray @MMLTouray}

Acknowledgements The authors acknowledge the trial funders Diabetes UK and all the patients and carers participating in the trial. The Centre for Trials Research receives funding by the Welsh Government through Health and Care Research Wales and the authors gratefully acknowledge the Centre's contribution to trial implementation. The authors acknowledge the contribution of the Trial Steering Committee (Adele McEvilly, Chris Patterson and Michael Bowdery), chaired by Dr Peter Swift; the trial administrator, Jackie Swain; the clinical teams at each of the 8 trial sites, the DECIDE project nurses and research nurses from NISCHR who provided support to the trial; the participating NHS Trusts and local Principal Investigators were The Royal Hospitals Belfast Health and Social Care Trust (Dr Dennis Carson), Cambridge (Dr Carlo Acerini), Cardiff and Vale NHS Trust (Professor J W Gregory and Dr J T Warner), Hull and East Yorkshire Hospitals NHS Trust (Dr Verghese Mathew), Alder Hey Children's NHS Foundation Trust, Liverpool (Dr Princy Paul), Newcastle upon Tyne Hospitals Foundation NHS Trust (Dr Tim Cheetham), Nottingham University Hospitals NHS Trust (Dr Tabitha Randell), University Hospital, Southampton NHS Trust (Dr Nicola Trevelyan and Dr Justin Davies); the stakeholders and others who have contributed. We pay tribute to Dr Carlo Acerini who sadly predeceased the publication of this report.

Contributors JWG and $L L$ are the joint chief investigators and guarantors of the trial in its entirety. LL, JWG, KH and MR were responsible for developing the research question and trial design and implementation of the trial protocol. NH and JT were responsible for trial and data management. TP and RP were responsible for all statistical considerations and analysis. LL, JWG and JT were responsible for drafting the manuscript. DC and ML supported by MT were responsible for designing, and together with TP for performing and reporting the resource use analysis. SC was responsible for advising on trial design. JD, TR, JW and NT were responsible for developing the research question and implementing the trial protocol. All those listed as authors contributed to trial delivery through the Trial Management Group and were responsible for reading, commenting on and approving the final manuscript. The manuscript's guarantors (JWG and LL) affirm that the manuscript is an honest, accurate and transparent account of the study being reported; that no important aspects of the study have been omitted and that any discrepancies from the study as planned (and, if relevant, registered) have been explained.

Funding This work was supported by Diabetes UK grant number RD06/0003353. Competing interests None declared.

Patient consent for publication Not required.

Provenance and peer review Not commissioned; externally peer reviewed.

Data availability statement Data are available on reasonable request.

Open access This is an open access article distributed in accordance with the Creative Commons Attribution Non Commercial (CC BY-NC 4.0) license, which permits others to distribute, remix, adapt, build upon this work non-commercially, and license their derivative works on different terms, provided the original work is properly cited, appropriate credit is given, any changes made indicated, and the use is non-commercial. See: http://creativecommons.org/licenses/by-nc/4.0/.

\section{ORCID iDs}

Julia Townson http://orcid.org/0000-0001-8679-3619

Michael Robling http://orcid.org/0000-0002-1004-036X 


\section{REFERENCES}

1 Royal College of Paediatrics and Child Health. National paediatric diabetes audit report, 2015 - 2016. Available: http://www.rcpch.ac. uk/improving-child-health/quality-improvement-and-clinical-audit/ national-paediatric-diabetes-audit-n-0\#2015-16

2 Landolt MA, Ribi K, Laimbacher J, et al. Posttraumatic stress disorder in parents of children with newly diagnosed type 1 diabetes. J Pediatr Psychol 2002;27:647-52.

3 Lowes L, Lyne P, Gregory JW. Childhood diabetes: parents' experience of home management and the first year following diagnosis. Diabet Med 2004;21:531-8.

4 House of Commons Health Committee. Managing the care of people with long-term conditions. Second Rep Sess 2014;:1:1-89.

5 Norris SL, Chowdhury FM, Van Le K, et al. Effectiveness of community health workers in the care of persons with diabetes. Diabet Med 2006;23:544-56.

6 Clar C, Waugh N, Thomas S, et al. Routine hospital admission versus out-patient or home care in children at diagnosis of type 1 diabetes mellitus. Cochrane Database Syst Rev 2007;31.

7 Lowes L, Gregory JW. Management of newly diagnosed diabetes: home or hospital? Arch Dis Child 2004;89:934-7.

8 Silverstein J, Klingensmith G, Copeland K, et al. Care of children and adolescents with type 1 diabetes: a statement of the American diabetes association. Diabetes Care 2005;28:186-212.

9 Dougherty GE, Soderstrom L, Schiffrin A. An economic evaluation of home care for children with newly diagnosed diabetes: results from a randomized controlled trial. Med Care 1998;36:586-98.

10 Charron-Prochownik D, Maihle T, Siminerio L, et al. Outpatient versus inpatient care of children newly diagnosed with IDDM. Diabetes Care 1997;20:657-60.

11 Chase HP, Crews KR, Garg S, et al. Outpatient management vs in-hospital management of children with new-onset diabetes. Clin Pediatr 1992;31:450-6.

12 Lee PD. An outpatient-focused program for childhood diabetes: design, implementation, and effectiveness. Tex Med 1992;88:64-8.

13 Swift PG, Hearnshaw JR, Botha JL, et al. A decade of diabetes: keeping children out of hospital. BMJ 1993;307:96-8.

14 Cowan FJ, Warner JT, Lowes LM, et al. Auditing paediatric diabetes care and the impact of a specialist nurse trained in paediatric diabetes. Arch Dis Child 1997;77:109-14

15 Simell T, Moren R, Keltikangas-Järvinen L, et al. Short-Term and longterm initial stay in hospital of children with insulin-dependent diabetes: adjustment of families after two years. Acta Paediatr 1995;84:41-50.

16 Townson JK, Gregory JW, Cohen D, et al. Delivering early care in diabetes evaluation (decide): a protocol for a randomised controlled trial to assess Hospital versus home management at diagnosis in childhood diabetes. BMC Pediatr 2011;11:7.

17 Ravens-Sieberer U, Bullinger M. Assessing health-related quality of life in chronically ill children with the German KINDL: first psychometric and content analytical results. Qual Life Res 1998;7:399-407.
18 Varni JW, Burwinkle TM, Jacobs JR, et al. The PedsQLTM in type 1 and type 2 diabetes: reliability and validity of the pediatric quality of life InventoryTM generic core scales and type 1 diabetes module. Diabetes Care 2003;26:631-7.

19 Kovacs M, lyengar S, Goldston D, et al. Psychological functioning of children with insulin-dependent diabetes mellitus: a longitudinal study. J Pediatr Psychol 1990;15:619-32.

20 Beeney LJ, Dunn SM, Welch M. Measurement of diabetes knowledge - the development of the DKN scales. In: Handbook of psychology and diabetes. UK Harwood, 1994.

21 Marteau TM, Bekker $\mathrm{H}$. The development of a six-item short-form of the state scale of the Spielberger State-Trait anxiety inventory (STAI). Br J Clin Psychol 1992;31:301-6.

22 Kovacs M, lyengar S, Goldston D, et al. Psychological functioning among mothers of children with insulin-dependent diabetes mellitus: a longitudinal study. J Consult Clin Psychol 1990;58:189-95.

23 National Glycohemoglobin Standardization Program. Harmonizing hemoglobin A1c testing: a better Test means better diabetes care. Standardization, International Federation of Clinical Chemistry, 2010. Available: http://www.ngsp.org/ifccngsp.asp [Accessed 8 Aug 2017].

24 Morgan-Trimmer S, Channon S, Gregory JW, et al. Family preferences for home or hospital care at diagnosis for children with diabetes in the decide study. Diabet Med 2016;33:119-24.

25 Arch BN, Blair J, McKay A, et al. Measurement of HbA1c in multicentre diabetes trials - should blood samples be tested locally or sent to a central laboratory: an agreement analysis. Trials 2016;17:517.

26 Tiberg I, Katarina SC, Carlsson A, et al. Children diagnosed with type 1 diabetes: a randomized controlled trial comparing Hospital versus home-based care. Acta Paediatr 2012;101:1069-73.

27 Clapin H, Hop L, Ritchie E, et al. Home-Based vs inpatient education for children newly diagnosed with type 1 diabetes. Pediatr Diabetes 2017;18:579-87.

28 Tiberg I, Lindgren B, Carlsson A, et al. Cost-effectiveness and cost-utility analyses of hospital-based home care compared to hospital-based care for children diagnosed with type 1 diabetes; a randomised controlled trial; results after two years' follow-up. BMC Pediatr 2016;16:1-12.

29 Jasinski CF, Rodriguez-Monguio R, Tonyushkina K, et al. Healthcare cost of type 1 diabetes mellitus in new-onset children in a hospital compared to an outpatient setting. BMC Pediatr 2013;13:55.

30 Mazarello Paes V, Charalampopoulos D, Edge J, et al. Predictors of glycemic control in the first year of diagnosis of childhood onset type 1 diabetes: a systematic review of quantitative evidence. Pediatr Diabetes 2018;19:18-26.

31 Beckles ZL, Edge JA, Mugglestone MA, et al. Diagnosis and management of diabetes in children and young people: summary of updated NICE guidance. BMJ 2016;352.

32 Randell T. Developing a best practice tariff in paediatric diabetes. Practical Diabetes 2012;29:176-7. 